Musées, Patrimoine et Culture scientifiques et techniques

$153 \mid 2014$

mai-juin 2014

\title{
Le récolement des collections entomologiques
}

François Dusoulier

URL : http://journals.openedition.org/ocim/1375

DOI : $10.4000 /$ ocim. 1375

ISSN : 2108-646X

Éditeur

OCIM

Édition imprimée

Date de publication : 25 juin 2014

ISSN : 0994-1908

Référence électronique

François Dusoulier, «Le récolement des collections entomologiques », La Lettre de I'OCIM [En ligne] 153 | 2014, mis en ligne le 25 juin 2016, consulté le 19 avril 2019. URL : http:// journals.openedition.org/ocim/1375; DOI : 10.4000/ocim.1375 


\section{Le récolement des collections entomologiques}

François Dusoulier *

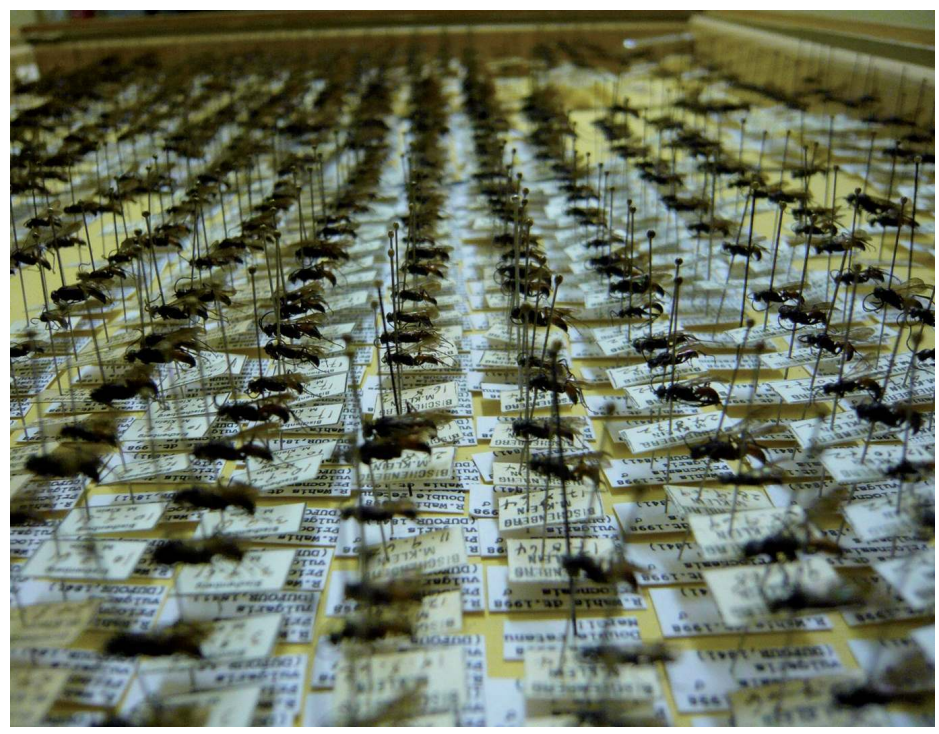

Compter les insectes dans les boîtes, pas si simple! ๑) Musée zoologique de Strasbourg/François Dusoulier.

François Dusoulier est conservateur du patrimoine, directeur du muséum d'Histoire naturelle de Toulon et du Var fdusoulier@cgvar.fr
Face à des collections d'insectes constituées de dizaines ou de centaines de spécimens, l'auteur s'interroge sur la manière de compter ces grands nombres, met en lumière les difficultés rencontrées et fournit quelques pistes qui allient harmonisation, rigueur et réalisme des tâches à accomplir.

Les collections d'insectes des muséums territoriaux représentent environ 8000000 de spécimens, soit approximativement $47 \%$ de la totalité des collections d'Histoire naturelle de France. Cette évaluation résulte de la compilation faites par Bertrand (2001) et Dusoulier (2004), revue et corrigée en 2013 en prenant en compte les nouvelles données du récolement échangées sur la liste de discussion nationale des personnels des muséums. Par ailleurs, le nombre d'insectes conservés au Muséum national d'Histoire naturelle (Paris) est estimé à 35 millions de spécimens. Il est probable que les deux seuls ordres des lépidoptères (papillons) et des coléoptères (scarabées, coccinelles...) concentrent l'essentiel de ces spécimens.

Matériellement, ces collections se présentent sous la forme de boîtes, vitrées ou non, rassemblant chacune des dizaines à des centaines de spécimens. Généralement acquises par les muséums sans inventaires précis, et exceptionnellement exposées au public du fait de la petite taille des spécimens qu'elles contiennent, ces collections ont rarement fait l'objet d'une attention soutenue. Pourtant, chacune constitue le témoignage unique d'un naturaliste - amateur ou professionnel - le fruit d'un travail d'une grande rigueur scientifique et technique, ainsi que de précieux matériaux de référence pour les sciences, datés et localisés. En effet, la valeur scientifique et patrimoniale d'une collection entomologique ne vient pas de l'insecte isolé, 
mais de l'étiquette qui l'accompagne et des informations qu'elle mentionne. Un travail pionnier de recherche en biohistoire a récemment démontré de manière brillante tout l'intérêt d'utiliser ces collections pour quantifier et qualifier l'érosion de la biodiversité (Perrein, 2012).

À l'instar des autres collections d'Histoire naturelle, les spécimens d'insectes constituent des objets de référence, notamment dans le domaine de la nomenclature, de la systématique et de la taxinomie. Toutefois, le grand nombre d'espèces d'insectes (plus de 40000 en France) et l'état très lacunaire des connaissances de certains groupes, font de ces collections des outils du quotidien pour les systématiciens et les naturalistes. Rappelons que même dans un pays comme la France, une très large majorité des espèces n'est toujours pas illustrée, que ce soit dans les ouvrages grand public ou spécialisés, ou même sur Internet. Laccès à ces collections par la communauté naturaliste est ainsi de première importance. Cet état de fait renforce clairement l'intérêt d'une bonne connaissance de ces collections au sein des muséums.

L'obligation du récolement décennal peut être ainsi envisagée comme une parfaite occasion pour s'intéresser de plus près à ces collections. Toutefois, trois difficultés majeures ont été identifiées pour la mise en œuvre méthodologique du récolement de ces collections entomologiques:

- l'inventaire des collections n'existe pas ou fait simplement état d'un nombre de boîtes et d'un nombre approximatif de spécimens ;

- le nombre d'objets est très différent suivant que l'on compte les contenants (boîtes ou couches), les spécimens et/ou leurs appendices annexes ;

- le marquage individuel est rendu malaisé par la taille et la diversité des spécimens.

\section{Il y a inventaire et inventaire !}

Dans la plupart des cas, les inventaires qui listent le contenu des collections d'insectes ne sont pas des inventaires réglementaires, au sens du Code du Patrimoine. Dans les meilleurs cas, ils décrivent les taxons traités et le nombre de boîtes. Comment s'assurer alors que tous les spécimens figurent bien dans chacune des boîtes inscrites à l'inventaire ? En effet, l'opération perd tout son sens si les boîtes sont présentes mais pas leur contenu! C'est la raison pour laquelle la loi prévoit de faire un renvoi à un sous-inventaire pour chaque lot complexe (boîte contenant les insectes). Cette tâche honorable représente néanmoins un travail titanesque puisque chaque muséum possède en moyenne 160000 spécimens d'insectes : en considérant la saisie de 12 spécimens par heure dans le sous-inventaire détaillé, cela nécessiterait le travail de 8,3 agents à plein temps pendant un an. Combien de muséums pourront tenir de tels engagements?

\section{Compter mais compter quoi ?}

La méthodologie du récolement s'accompagne de difficultés dès lors que l'on s'attache à compter les objets au sein des lots et ensembles complexes. Il est alors difficile de s'en tenir à la règle édictant qu'un objet doit/devrait correspondre à un numéro d'inventaire. La complexité est induite par le grand nombre de spécimens concentrés dans chaque boîte ; les insectes sont présentés piqués sur épingles ou minuties, collés sur paillette, ou plus sommairement fixés sur du coton dans des « couches ». Il y a très souvent des parties de spécimens qui tombent au fond des boîtes, parfois même des individus entiers. Comment prendre cela en compte dans le récolement? Chez les petites espèces, on observe régulièrement plusieurs paillettes posées en étage sur une même épingle ; cette présentation dite en « HLM » ou en « logements sociaux » est rarement considérée. Pourtant, elle peut influencer significativement le comptage des individus. Par ailleurs, elle apporte potentiellement des spécimens non conspécifiques qui viennent enrichir la collection. Pour l'expert entomologiste, l'examen de ces spécimens sous-observés permet parfois de belles découvertes.

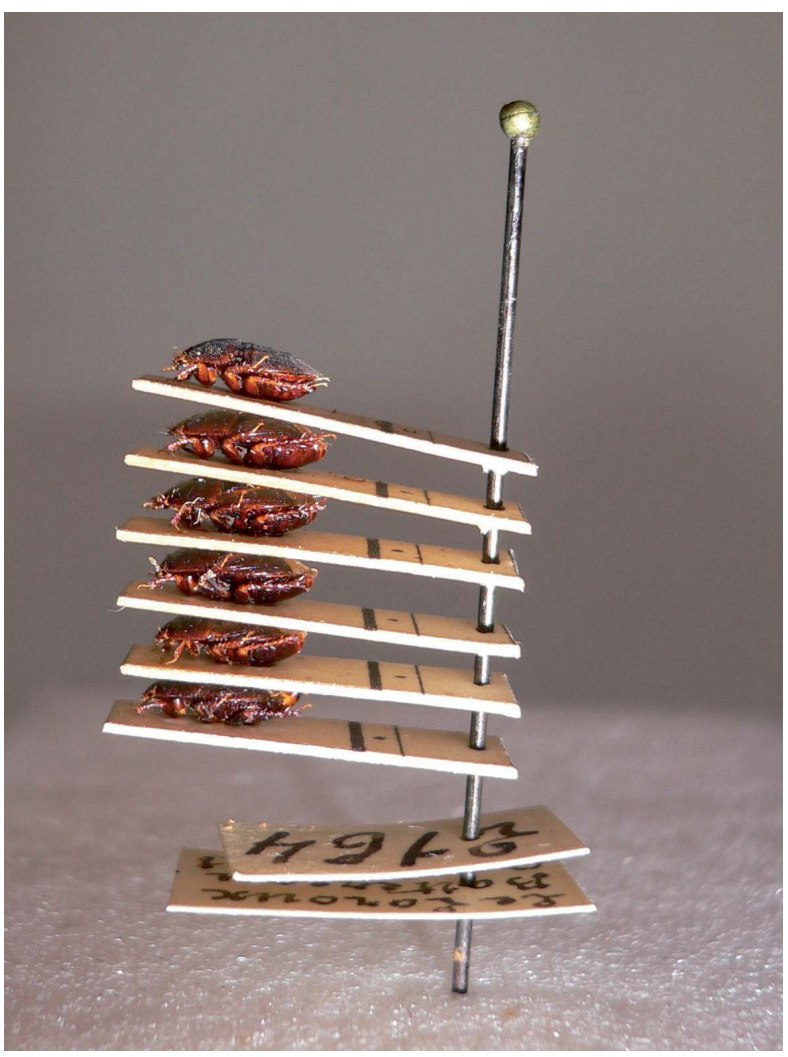

Des punaises Cydnidae en « logements sociaux » sur une épingle (c) Muséum d'Histoire naturelle de Nantes/François Dusoulier 
Le cas des génitalia mérite d'être abordé tant ils sont des caractères utiles dans la classification et la détermination des espèces d'insectes. Comme pour les collections ostéologiques, le comptage de ces préparations nous donne un nombre d'objets supérieur au nombre de spécimens. Parfois, ces organes sont directement collés sur la paillette ou encore mis dans un tube dont le bouchon est piqué sous le spécimen. La comptabilisation de ces pièces annexes est alors facilitée. À la façon des pièces ostéologiques des vertébrés, il faudrait éventuellement les considérer comme des sous-ensembles des spécimens...

\section{Un marquage délicat}

Le marquage individuel est illusoire, tant par la taille et le nombre des spécimens que pour ne pas porter atteinte à l'intégrité de la présentation de la collection. Lorganisation d'une collection d'insectes est en effet un révélateur fort de la culture et de la personnalité de celui qui l'a constituée

Évidemment, le soin particulier apporté à la disposition et à la préparation technique des spécimens varie beaucoup ; dans la plupart des cas, c'est la boîte qui fait œuvre scientifique et patrimoniale, très rarement le spécimen.

L'une des exceptions est celle des onomatophores (spécimens porte-noms), plus communément appelés «types ». Ces individus ont un rôle d'interface particulier, puisque leur désignation par un systématicien leur permet d'assurer le lien matériel entre le monde du vivant et le monde du langage humain. Leur valeur patrimoniale comme objet irremplaçable permettant la description de la biodiversité est incommensurable. Leur inventaire précis devrait ainsi être effectué prioritairement au sein de chaque unité d'inventaire. Chacun d'entre eux pourrait éventuellement recevoir un numéro propre. Hélas, leur identification est délicate, notamment dans les collections antérieures aux règles édictées par les différentes éditions du Code International de Nomenclature Zoologique (ICZN, 1999).

\section{Comment faire ?}

Le récolement des collections entomologiques doit répondre à deux logiques : une certaine harmonisation

\section{l'expérience du musée des Papillons}

La collection entomologique du musée des Papillons de Saint-Quentin était estimée à 600000 spécimens. Les inventaires retrouvés (simple dénombrement des boîtes) n'étaient pas réglementaires. Sans inventaire par spécimen, l'option retenue est de considérer 1 boîte = 1 lot $=1$ objet avec son numéro. 1348 lots doivent ainsi être récolés. En 2011, 10 jours de récolement permettent de saisir 189 lots informatiquement. En 2012 (fermeture temporaire et 2 agents à disposition), 796 lots rejoignent la base (moyenne 30 boites/jour). 94 lots supplémentaires sont récolés en 2013 , atteignant $80 \%$ de l'objectif global. La principale difficulté d'une opération de récolement est de la mener en parallèle des autres activités culturelles du musée.

Cependant, le récolement a été l'occasion de dénombrer les spécimens présents par boîte, ce qui nous permet d'avoir une vue nettement plus précise de la taille des collections. Ainsi, sur 600000 spécimens estimés depuis plus d'un siècle, nous arrivons actuellement à 87058 spécimens pour $80 \%$ des unités estimées à récoler. Le chiffre définitif approchera probablement la centaine de milliers ce qui semble plus raisonnable au regard du nombre de boîtes présentes. La taille très variable des spécimens influe nécessairement sur la composition des boîtes, et si une moyenne est possible (94 spécimens/boîte actuellement) elle ne peut apporter de véritable information en raison d'une grande disparité (de 1 à 631 spécimens/boîte !).

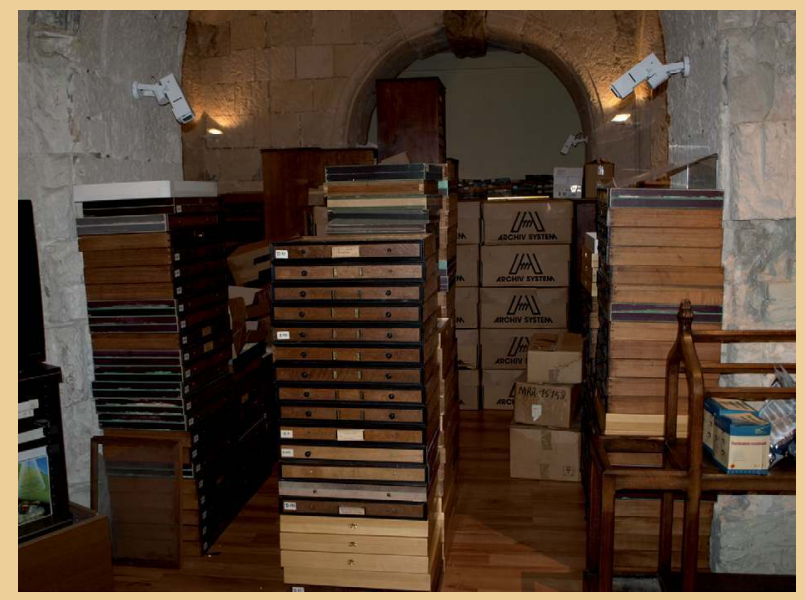

Stockage des collections dans l'exposition permanente à l'occasion de la rénovation de la réserve (automne-hiver 2011-2012) ○) Musée des Papillons/Agnès Villain

\section{Agnès Villain} Attachée de conservation du Patrimoine (PSTN), directrice du musée des Papillons 
des pratiques et un certain réalisme pour le travail. Pour chaque objet (le plus souvent une boîte), l'idéal serait :

- d'y inscrire un numéro d'inventaire unique ;

- de faire une photographie de référence de l'ensemble ; - de décrire le contenu taxinomique supra-spécifique (ordre, familles, genres), la provenance par grand domaine biogéographique, le dénombrement précis des spécimens, l'état de conservation général et les éventuelles altérations et le repérage des onomatophores. Ce long travail permettra seul de dresser un point initial de l'état de conservation et de la diversité des collections des muséums en matière d'entomologie. Souhaitons qu'en portant à la connaissance de chacun de tels instruments de recherche, des chantiers dédiés à l'étude précise et détaillée de ce biopatrimoine puissent être mis en œuvre.

\section{Bibliographie}

Bertrand, R. Les collections des muséums d'Histoire naturelle en France. Rapport dactylographié du ministère de la Recherche, direction de la Recherche, mission de la culture et de l'information scientifiques et techniques et des musées, 2001, 55 p.

Dusoulier, F. Muséum d'Histoire naturelle de la ville d'Aix-en-Provence (13): les collections entomologiques et paléo-entomologiques. Mémoire pour la validation du travail de recherche à l'Institut national du patrimoine, Paris, 2004, $121 \mathrm{p}$.

ICZN International Code of Zoological Nomenclature. London : The International Trust for Zoological Nomenclature, Fourth edition, 1999, 306 p.

Perrein, C. Biohistoire des papillons. Diversité et conservation des lépidoptères rhopalocères en Loire-Atlantique et en Vendée. Rennes : Presses universitaires de Rennes, 2012, 621 p. 